\title{
Gain-clamped double-pass S-band erbium-doped fiber amplifier
}

\author{
Sulaiman Wadi Harun ${ }^{1 a)}$, Muhammad Sabri Abdul Rahman², \\ and Harith Ahmad ${ }^{2}$ \\ ${ }^{1}$ Faculty of Engineering, Multimedia University, 63100 Cyberjaya Selangor, \\ Malaysia. \\ 2 Photonics Laboratory, Department of Physics, University of Malaya \\ 50603 Kuala Lumpur, Malaysia \\ a)wadi72@yahoo.com
}

\begin{abstract}
Gain clamping in double-pass short-wavelength band erbium-doped fiber amplifier (S-band EDFA) is demonstrated. It uses a fiber Bragg grating, which operates in conventional-band to form an oscillating laser in the cavity using an erbium-doped fiber (EDF) gain at around $1530 \mathrm{~nm}$. This new technique has shown a good gain clamping effect with gain variation of less than $1.0 \mathrm{~dB}$ from -40 to $-14 \mathrm{dBm}$ input signal powers for $1500 \mathrm{~nm}$ signal. The gain can be controlled within 18 to $20 \mathrm{~dB}$ by varying a variable optical attenuator from 0 to $8 \mathrm{~dB}$. Compared with an unclamped amplifier, the noise figures for small signals are slightly improved due to the suppression of amplified spontaneous emission (ASE). However, the noise figure level is still considerably high due to the double-pass scheme and unoptimised splicing between a depressed cladding EDF and a standard single mode fiber. The advantage of this gain clamped amplifier is that the oscillating light operates at wavelength outside S-band region, which prevent the wavelength division multiplexed system from being disturbed.
\end{abstract}

Keywords: S-band EDFA, depressed cladding EDF, Erbium-doped fiber amplifier, double-pass EDFA, gain clamping

Classification: Photonics devices, circuits, and systems

\section{References}

[1] S. W. Harun, N. K. Saat, and H. Ahmad, "An Efficient S-Band ErbiumDoped Fiber Amplifier Using Double-Pass Configuration," IEICE Electron. Express, vol. 2, no. 6, pp. 182-185, 2005.

[2] M. Zirngibl, "Gain control in erbium-doped fiber amplifiers by an alloptical feedback loop," Electron. Lett., vol. 27, no. 7, pp. 560-561, 1991.

[3] T. Subramaniam, M. A. Mahdi, P. Poopalan, S. W. Harun, and H. Ahmad, "All-optical gain-clamped erbium-doped fiber ring lasing amplifier with laser filtering technique," IEEE Photon. Technol. Lett., vol. 13, pp. 785$787,2001$.

[4] S. W. Harun, N. Tamchek, P. Poopalan, and H. Ahmad, "Gain control in L-band erbium-doped fiber amplifier using a ring resonator," Jpn. J. 
Appl. Phys., vol. 41 (3B), Part 2, pp. L332-L333, 2002.

[5] P. C. Becker, N. A. Olsson, and J. R. Simpson, "Erbium-Doped Fiber Amplifier - Fundamentals and Technology," Academic Press, San Diego USA, 1999.

[6] Y. Zhao, J. Bryce, and R. Minasian, "Gain clamped erbium-doped fiber amplifiers-modeling and experiment," IEEE J. Select. Topics Quantum Electron., vol. 3, pp. 1008-1012, 1997.

[7] E. Desurvire, "Erbium-Doped Fiber Amplifier- principle and applications," John Wiley and Sons, Inc. New York, 1994.

\section{Introduction}

Short wavelength band erbium-doped fiber amplifiers (S-band EDFAs) for wavelength division multiplexed (WDM) transmission system are very attractive because the transmission capacity in conventional band (C-band) and long wavelength band (L-band) is gradually becoming insufficient and S-band is a potential candidate to extend the capacity by all means. Recently, a double-pass configuration have been proposed in order to improve the gain performance of the S-band EDFA [1]. The double-pass amplifier requires fewer components than the conventional two-stage bi-directional pumped configurations, which make it more cost-effective than the conventional single-pass amplifier. However, gain clamped characteristics are indispensable in the EDFAs for the reason that in WDM systems, the channel gains are required to be constant even though the operating conditions, such as the total input signal power and pump power, can vary significantly. The transient response is another issue that needs to be considered as large dynamic excursion can lead to errors at the receiver and degrades system performance. Therefore, the gain clamping techniques have been extensively explored for C- and L-band EDFAs to control the dynamic gain variation, including the use of tunable bandpass filter and fiber Bragg grating (FBG) to form laser in optical feedback loop $[2,3,4]$. However, there are still a lack of research work on the gain-clamped S-band EDFA. In this letter, we propose a simple and novel S-band gain-clamped EDFA using a FBG in double-pass configuration.

\section{Experimental set up}

The configuration of the double-pass S-band EDFA is shown in Fig. 1. A $15 \mathrm{~m}$ depressed cladding EDF is used to provide a high distributed loss at wavelength longer than $1525 \mathrm{~nm}$ for S-band amplification. The composition of the EDF's core is approximately $2.5 \% \mathrm{GeO}_{2}, 5.5 \% \mathrm{Al}_{2} \mathrm{O}_{3}$, and $92 \% \mathrm{SiO}_{2}$ with 0.15 wt.\% Erbium. The depressed cladding is approximately $3 \%$ Fluorine, $0.5 \% \mathrm{P}_{2} \mathrm{O}_{5}$, and $96.5 \% \mathrm{SiO}_{2}$. The numerical aperture of the core, relative to the depressed cladding, is 0.22 . The background loss of EDF is less than $5 \mathrm{~dB} / \mathrm{Km}$. The EDF is pumped by $980 \mathrm{~nm}$ laser diode using a forward pumping scheme and the pump absorption is about $7.6 \mathrm{~dB} / \mathrm{m}$. A 


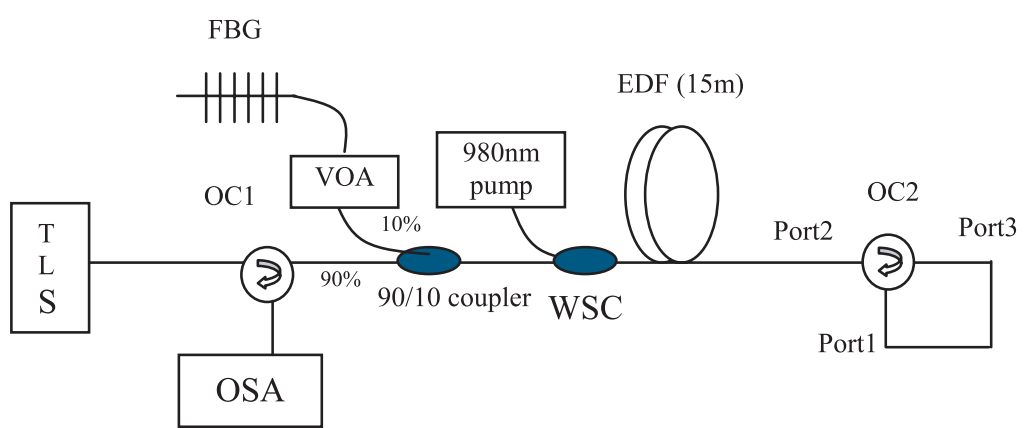

Fig. 1. Configuration of the proposed double-pass gainclamped S-band EDFA.

wavelength selective coupler (WSC) optimized for C-band operation is used to combine the $980 \mathrm{~nm}$ pump with the test signal. An optical circulator OC2 with port 1 connected to port 3 is employed at the output end of the EDF to allow double propagation of test signal in the system. Optical circulator OC1 is placed at the input end of the amplifier to route the amplified signal into an optical spectrum analyzer (OSA). A 90/10 coupler is used in conjunction with a broadband FBG to form an oscillating laser in the cavity for gain clamping. The broadband FBG has a reflectivity of about $99 \%$ and a bandwidth of $40 \mathrm{~nm}$ centered at $1545 \mathrm{~nm}$. It reflects the backward ASE of wavelength around $1530 \mathrm{~nm}$ back into the system to oscillate in the cavity and thus clamps the gain. A variable optical attenuator (VOA) is inserted to control the oscillating light in the cavity. A tunable laser source (TLS) is used for evaluation of the amplifier performances in conjunction with an OSA.

\section{Result and discussion}

The gain and noise figure characteristics as functions of input signal power for the gain clamped amplifier is shown in Fig. 2. Measurements of the gain and noise figure were performed at different input signal power levels, from -40 to $4 \mathrm{dBm}$ at $1500 \mathrm{~nm}$. The pump power is fixed at $103 \mathrm{~mW}$. For the amplifier with VOA close, the small signal gain was obtained at $26 \mathrm{~dB}$ and degrades as the input signal power increases. On the other hand, gains for the amplifier with VOA open are clamped at small input signals. For instance, at VOA = $2 \mathrm{~dB}$, the gains are clamped at about $20.0 \mathrm{~dB}$ with gain variation of less than $1.0 \mathrm{~dB}$, for all input signal powers from -40 to $-14 \mathrm{dBm}$. This gain clamping is due to the incorporation of the FBG, which reflects the ASE of wavelengths above $1525 \mathrm{~nm}$. This will create an oscillating laser at wavelength around $1530 \mathrm{~nm}$ between the FBG and OC2. The laser operates at this wavelength due to the C-band ASE level is highest at this region. This oscillating laser fixed the average population inversion by extracting the energy stored in the amplifier due to the homogeneous broadening [5]. In a homogeneously broadened medium, the lasing action fixes the total population inversion. Any variation in other conditions, such as the input signal power, would be compensated by the adjustment of the lasing power [6]. This is especially 


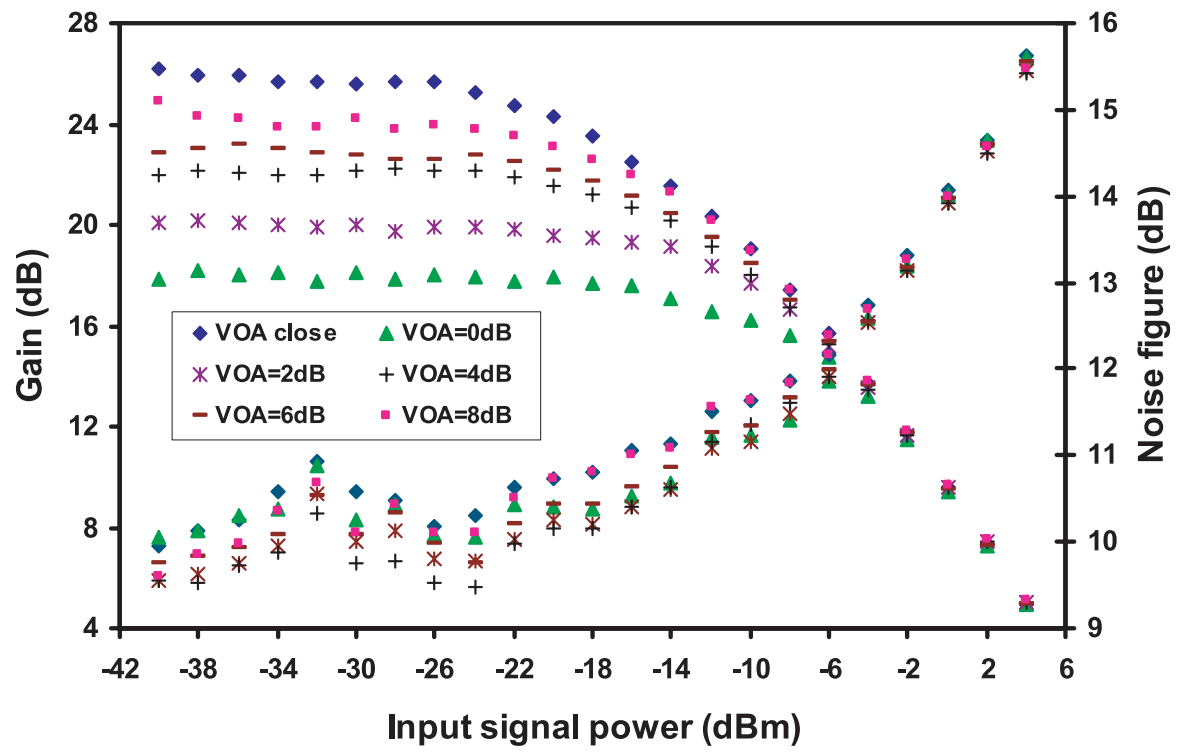

Fig. 2. The measured gain and noise figure against input signal power at various VOA settings.

true when the input signal power is low (lower than the laser output power level) as shown in Fig. 2. However, as the input signal power is increased, the laser power is decreased and becomes extinguished hence the gain is no longer clamped. The clamped gain can also be controlled within 18 to $24 \mathrm{~dB}$ by varying the VOA from 0 to $8 \mathrm{~dB}$ as shown in Fig. 2. The gain variation under gain clamping is less than $1 \mathrm{~dB}$ and it is generated due to the inhomogeneous gain medium. This variation, if high, will cause transient response, which in turn increases bit error rate of the system.

On the other hand, noise figures measured for the gain clamped amplifier are about 9.5 to $11.0 \mathrm{~dB}$ in the gain clamped region, which is slightly better compared to that of the case with VOA close. These values are relatively high due to the insertion loss of the 90/10 WDM coupler and unoptimised splicing between the depressed cladding EDF and the standard single-mode fiber, which is estimated to be more than $1 \mathrm{~dB}$. The double-pass scheme also contributes to the noise figure due to the double-propagation of signal in the EDF, which reduces the population inversion at the input part of the amplifier. The low population inversion contributes to higher noise in the input end and the noise is amplified in the amplifier, which resulted in the higher overall noise figure. The noise figure of the gain clamped amplifier is lower than that of the unclamped amplifier (VOA close), because the laser power compressed the ASE power strongly and the operation point of the amplifier fell in the dip region of the original noise profile [7]. Fig. 2 also shows the noise figure curve has a dip at $-26 \mathrm{dBm}$ input signal power region, which is attributed to the interplay between self-saturation by backward ASE and self-induced saturation [7].

The gain and noise figure spectra of the unclamped and clamped amplifier are shown in Fig. 3. The input signal and pump powers are fixed at $-30 \mathrm{dBm}$ 


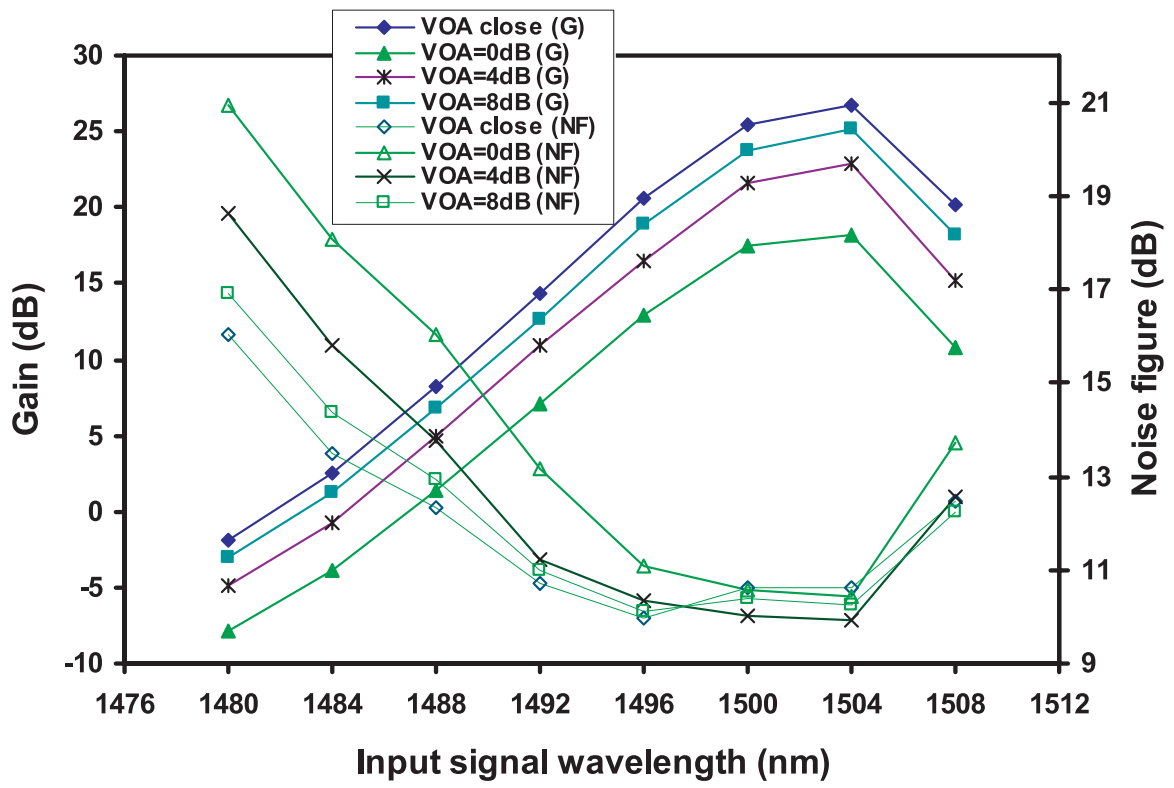

Fig. 3. The gain and noise figure spectra at various VOA settings.

and $103 \mathrm{~mW}$, respectively. The gains for the gain clamped amplifier are reduced due to the laser extracting the energy stored in the amplifier. At wavelength below $1500 \mathrm{~nm}$, the gain clamped amplifier shows a higher noise figure compared to that of unclamped amplifier (VOA close) as shown in Fig. 3. This is attributed to the ASE compression at these wavelengths, which is very small because they are located far away from the oscillating laser. The lower gain also contributes to the higher noise figure at this region. The advantage of the both gain clamped system is that the oscillating laser does not appear at the output port since it operates at wavelength of around $1530 \mathrm{~nm}$, which is outside of S-band region. This prevents the WDM system from being disturbed by the oscillating laser.

\section{Conclusion}

A gain clamping is demonstrated in double-pass S-band EDFA by incorporating of FBG. The FBG reflects the C-band ASE to oscillate in the cavity between the FBG and $\mathrm{OC} 2$ for gain clamping. The clamped gain can be controlled to be within 18 to $24 \mathrm{~dB}$ by varying the VOA from 0 to $8 \mathrm{~dB}$. At $\mathrm{VOA}=2 \mathrm{~dB}$, the gain curves for the amplifier are clamped at about $20.0 \mathrm{~dB}$, for all input signal powers from -40 to $-14 \mathrm{dBm}$ with gain variation of less than $1.0 \mathrm{~dB}$. The corresponding noise figure is slightly improved compared with an unclamped amplifier. However, the noise figure level is still considerably high due to the double-pass scheme and unoptimised splicing. The advantage of this gain clamped scheme is that the oscillating light operates at C-band region and does not disturb the operation of WDM system. 